\title{
AMPLIFICATION OF ULTRAWIDEBAND SIGNALS
}

\author{
Won Namgoong, Jongrit Lerdworatawee \\ Department of Electrical Engineering \\ University of Southern California \\ namgoong@usc.edu, lerdwora@usc.edu
}

\begin{abstract}
One of the main implementation challenges in the ultrawideband (UWB) radio is the design of efficient amplifiers. The difficulty in amplifying an UWB signal stems from its bandwidth being a large fraction of the amplifier gain-bandwidth product. This paper describes a methodology and the tradeoffs associated with the design of UWB amplifiers. The amplifiers are designed to minimize a new performance metric, which we refer to as the effective noise figure (NF). The effective NF measures the degradation caused by the amplifier in the achievable receiver performance after the digital decoding process, which is ultimately the most relevant measure of performance.
\end{abstract}

\section{INTRODUCTION}

The ultrawideband (UWB) radio is a relatively new technology that is being pursued for both commercial and military purposes [1][2]. The rationale for deploying the UWB radio lies in the benefits of exceptionally wide bandwidths, thereby achieving a combination of very fine time/range resolution, high data rates, robustness to narrowband interferers, and ability to resolve multipath components [3].

One of the main implementation challenges in the UWB radio is the design of efficient UWB amplifiers. The difficulty arises from the finite gain-bandwidth product of most existing amplifiers. Since the UWB signal bandwidth is a large fraction of the gain-bandwidth product, achieving a reasonable gain with enough bandwidth to pass the wideband received signal largely undistorted is difficult.

The performance of an amplifier is generally quantified using the noise factor (or noise figure in $\mathrm{dB}$ ), which is defined as the ratio of the signal-to-noise ratio (SNR) at the input of the amplifier to the SNR at the output of the amplifier. Although the use of the noise figure (NF) metric is straightforward in narrowband systems, its use becomes more difficult in UWB systems. The main difficulty arises in defining the SNR. In a narrowband system, where both the input signal and noise are assumed to be a single tone at the carrier frequency, the SNR is obtained by simply dividing the signal power by the noise power. In an UWB system, however, the input signal is broadband and the additive noise may be colored. The SNR obtained by simply dividing the signal power by the total noise power (whose bandwidth must also be defined) is less meaningful,

This work was supported in part by the Army Research Office under contract number DAAD19-01-1-0477 and National Science Foundation under contract number ECS-0134629.

Permission to make digital or hard copies of all or part of this work for personal or classroom use is granted without fee provided that copies are not made or distributed for profit or commercial advantage and that copies bear this notice and the full citation on the first page. To copy otherwise, to republish, to post on servers or to redistribute to lists, requires prior specific permission and/or a fee.

ICCAD'03, November 11-13, 2003, San Jose, California, USA.

Copyright 2003 ACM 1-58113-762-1/03/0011 ...\$5.00. since a higher SNR value defined in this manner does not necessarily translate to a higher receiver performance. This is because the performance of the receiver after the digital decoding process does not depend on the total signal and noise power but on the power spectrum density (PSD) of the additive noise and the impulse responses of the propagation channel and the transmit pulse.

Because of the difficulty in defining the SNR, existing work on broadband amplifier defines the NF as the weighted average of the single-tone NF [4]. Although such definition of NF is an extension of a single-tone NF, minimizing such arbitrary performance metric does not necessarily improve the overall receiver performance.

For the NF of the amplifier to be a meaningful metric in an UWB receiver, the SNR at the input and output of the amplifier should measure the achievable performance after the eventual digital decoding process, as it is ultimately the most relevant measure of performance. Hence, we define the SNR as the matched filter bound (MFB) [5], which represents an upper limit on the performance of data transmission systems. The MFB is obtained when a noise whitened matched filter is employed to receive a single transmitted pulse. By defining the SNR as the MFB, the NF measures the degree of degradation in the achievable receiver performance caused by the amplifier. We subsequently refer to this NF as the effective NF.

This paper describes a methodology and the tradeoffs associated with the design of UWB amplifiers with a finite gain-bandwidth product. The amplifiers are designed by minimizing the effective NF, so that the performance after the digital signal processing is maximized. For ease of explanation, we assume a baseband UWB signal with bandwidth $B$, although similar analysis and conclusions can be made for modulated UWB signals.

The organization of this paper is as follows. Section 2 introduces the effective noise figure. The optimization of a single-stage amplifier is described in Section 3, and the cascaded stages in Section 4. Conclusions are drawn in Section 5.

\section{THE EFFECTIVE NOISE FIGURE}

A general system model of a communication channel including the amplifier is shown in Fig. 1(a). The $k$ th transmit symbol $x_{k}$ is filtered by the equivalent pulse response then corrupted by the additive noise $n_{i}(t)$. The equivalent pulse response (whose frequency response is $P(f)$ ) represents the combination of both the transmit pulse and the propagation channel. The resulting corrupted signal is the input to the amplifier, which has a transfer function given by $\left(G^{1 / 2}(f)\right)$ and internally additive noise $n_{g}(t)$ 


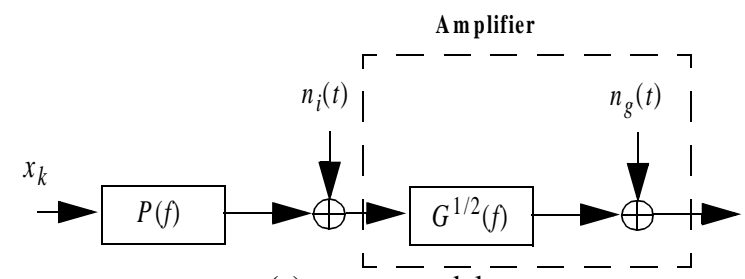

(a) system model

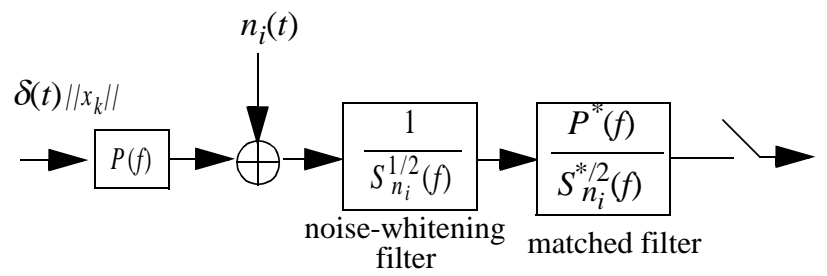

(b) input effective NF

Fig. 1. General communication channel model.

The MFB, also called the "one-shot" bound, is an upper limit on the performance of data transmission systems with intersymbol interference (ISI). As an example, the computation of the MFB at the input of the amplifier is illustrated in Fig. 1(b). An impulse is transmitted through the equivalent pulse response, which is then corrupted by $n_{i}(t)$. The input to the receiving system is noise whitened followed by a matched filter that is matched to both the pulse response and the noise whitening filter. $S_{n_{i}}(f)$ represents the PSD of $n_{i}(t)$. The matched filter output is then sampled when the output signal is at its maximum. The resulting SNR is the MFB.

The MFB at the input and output of the receiving system is [5]

$$
\begin{gathered}
S N R_{\text {in }}=\int \frac{|P(f)|^{2}}{S_{n_{i}}(f)} d f \\
S N R_{\text {out }}=\int \frac{|P(f)|^{2} G(f)}{S_{n_{i}}(f) G(f)+S_{n_{g}}(f)} d f
\end{gathered}
$$

Assuming, as is commonly done, that the input noise $n_{i}(t)$ is white with a PSD of $-174 \mathrm{dBm} / \mathrm{Hz}$, the effective NF of the receiving system can be written as a function of the spot NF by dividing (2) from (1) then rearranging, i.e.,

$$
F_{e f f}=\frac{1}{\int\left(\frac{|P(f)|^{2}}{P_{T}}\right) \frac{1}{F_{s}(f)} d f}
$$

where $P_{T}=\int|P(f)|^{2} d f$ and $F_{S}(f)$ denotes the spot NF as given by

$$
F_{s}(f)=\frac{S_{n_{i}}(f) G(f)+S_{n_{g}}(f)}{S_{n_{i}}(f) G(f)} .
$$

For a cascade of the multiple-stage receiving systems, $F_{s}(f)$ can be determined by the well-known Friis formula [6] i.e.,

$$
F_{s}(f)=F_{s 1}(f)+\frac{F_{s 2}(f)-1}{G_{1}(f)}+\ldots+\frac{F_{s N}(f)-1}{G_{1}(f) \ldots G_{N-1}(f)}
$$

where $F_{s i}(f)$ and $G_{i}(f)$ denote the spot NF and gain of the $i$ th cascaded receiving system.

To simplify the effective NF measurement, we assume that $P(f)$ is constant over the frequency band of interest. This is a reasonable assumption since the uncertainty in the propagation channel response makes the pulse response $P(f)$ generally unknown at design time. The effective NF given in (3) can then be approximated as

$$
F_{e f f} \approx \frac{N}{\sum_{i=0}^{N-1} \frac{1}{F_{s}\left(f_{i}\right)}}
$$

where $\left\{f_{0}, f_{1}, \ldots, f_{N-1}\right\}$ represent the center frequencies for each of the spot NF measurements in the frequency band of interest and $N$ is the total number of measured values. The effective NF equation in (6) is analogous to computing the normalized effective resistance of $N$ parallel resistors each with resistance $N \cdot F_{s}\left(f_{i}\right)$.

\section{SINGLE-STAGE AMPLIFIER}

An amplifier can be accurately modeled using the general model of the receiving system in Fig. 1(a). The following assumptions are made: $P(f)$ is an ideal brickwall filter with bandwidth $B$, which represents the bandwidth of the UWB signal; the input noise $n_{i}(t)$ and the internally generated noise $n_{g}(t)$ are both white with PSD $N_{i}$ and $N_{g}$, respectively; and the amplifier power gain is

$$
G(f)=\frac{K^{2}}{f^{2}+f_{3 d B}^{2}}
$$

where $K$ denotes the gain-bandwidth product (GBP) and $f_{3 d B}$ is the $3 \mathrm{~dB}$ bandwidth of the amplifier.

Substituting (7) into (4), the spot NF can be determined after straightforward algebraic manipulations as

$$
F_{s}(f)=1+\frac{N_{g}}{K^{2} N_{i}}\left[f^{2}+f_{3 d B}^{2}\right]
$$

As evident in (8), the spot NF is minimized at all freqencies by making $f_{3 d B}$ as small as possible. This occurs because the power gain given in (7) increases as $f_{3 d B}$ is decreased. The larger power gain suppresses the effects of the internally generated noise $n_{g}(t)$, which in turn results in smaller spot NF values.

The effective NF is readily determined by substituting (8) into (3) and integrating:

$$
F_{e f f}=\frac{\sqrt{F_{s}(0) \frac{N_{g}}{N_{i}} \frac{B^{2}}{K^{2}}}}{\operatorname{atan}\left\{\sqrt{\left.\frac{1}{F_{s}(0)} \frac{N_{g}}{N_{i}} \frac{B^{2}}{K^{2}}\right\}}\right.}
$$




$$
\equiv \frac{\sqrt{\gamma+\gamma^{2}\left(\frac{f_{3 d B}}{B}\right)^{2}}}{\operatorname{atan}\left\{\sqrt{\frac{\gamma}{1+\gamma\left(\frac{f_{3 d B}}{B}\right)^{2}}}\right\}}
$$

where $F_{s}(0)$ is the spot NF in (8) when $f=0$, and $\gamma$ is defined as $N_{g} B^{2} /\left(N_{i} K^{2}\right)$. Note that $F_{e f f}$ is fully characterized by $f_{3 d B} / B$ and $\gamma$. In Fig. 2, the effective NF (in $\mathrm{dB}$ ) is determined as a function of $f_{3 d B} / B$ for different $\gamma$ values. The effective NF improves as $f_{3 d B} / B$ is reduced, suggesting that the best performance is achieved by making the amplifier as narrowband as possible. This result is expected since, as described above, the spot NF is minimized at all frequencies by reducing $f_{3 d B}$. However, the drawback of employing a small $f_{3 d B}$ is that the signal bandwidth is also greatly reduced by the amplifier, which in turn diminishes some of the benefits of the UWB radio, such as the ability to resolve multipaths. As a compromise between these conflicting objectives, $f_{3 d B}$ corresponding to an effective NF that is slightly greater (e.g., $1 \mathrm{~dB}$ ) than the minimum effective NF can be chosen for a given $\gamma$ value.

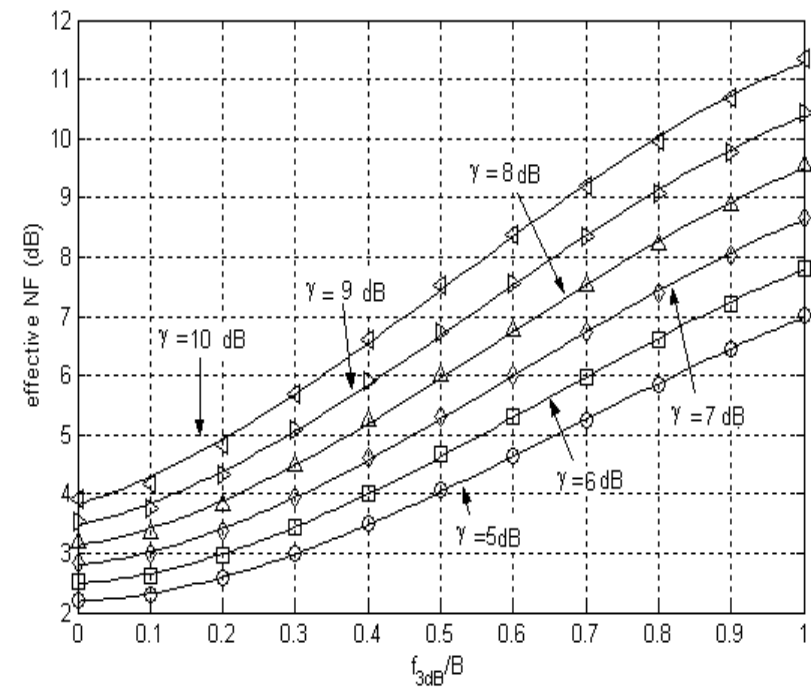

Fig. 2. The effective NF relating to $f_{3 d B} / B$ and $\gamma$ for the single amplifier sytem.

\section{CASCADED AMPLIFIER STAGES}

A system model of $M$ cascaded amplifier stages is shown in Fig. 3. $G_{l}(f)$ denotes the power gain of the $l$-th amplifier stage, where $l \in\{1,2, \ldots, M\}$, and $n_{g l}(t)$ is the internally generated noise of the $l$ th amplifier stage, whose PSD is assumed white and denoted as $N_{g l}$. The total power gain is

$$
G(f)=\prod_{l=1}^{M} \frac{K_{l}^{2}}{f^{2}+f_{l}^{2}},
$$

where $K_{l}$ and $f_{l}$ denote respectively the GBP and the $3 \mathrm{~dB}$ bandwidth of the $l$ th amplifier.

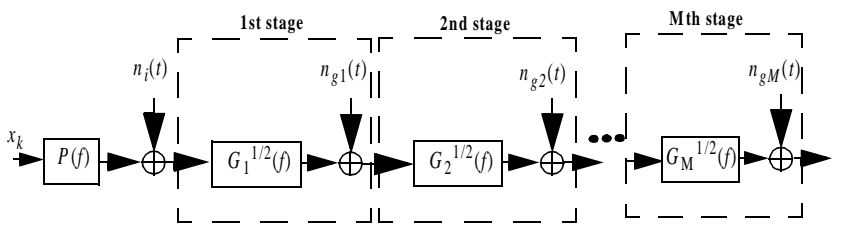

Fig. 3. M-stage amplifier system model.

By definition, the effective $f_{3 d B}$ of the $M$-stage amplifier can be obtain by solving the following implicit equation

$$
\prod_{l=1}^{M}\left[f_{3 d B}^{2}+f_{l}^{2}\right]=2 \prod_{i=l}^{M} f_{l}^{2} .
$$

Substituting (11) into (5), the spot NF of the $M$-stage amplifier becomes

$$
\begin{gathered}
F_{s}(f)=1+\gamma_{1}\left[f^{2}+f_{1}^{2}\right]+\gamma_{2}\left[f^{2}+f_{1}^{2}\right]\left[f^{2}+f_{2}^{2}\right]+\ldots, \\
+\gamma_{M} \prod_{l=1}^{M}\left[f^{2}+f_{l}^{2}\right]
\end{gathered}
$$

where

$$
\gamma_{l} \equiv \frac{N_{g l}}{N_{i}} \prod_{j=1}^{l} \frac{B^{2}}{K_{j}^{2}}, \quad l \in\{1,2, \ldots, M\}
$$

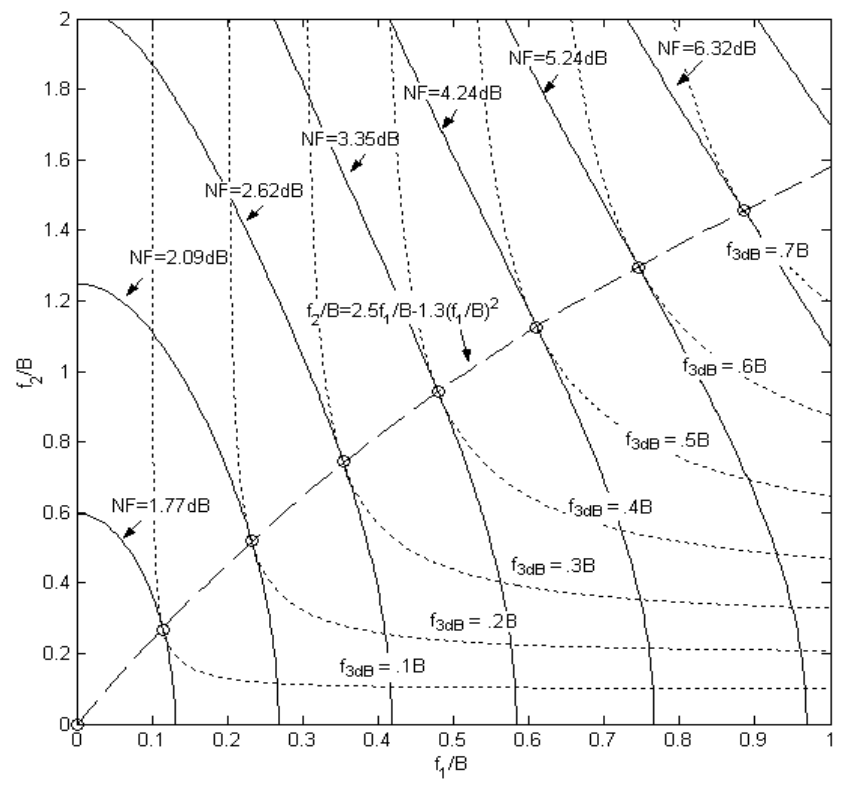

Fig. 4. Contours of effective $\mathrm{NF}$ (solid line) and $\mathrm{f}_{3 \mathrm{~dB}}$ (dash line) as a function of $\mathrm{f}_{1} / \mathrm{B}$ and $\mathrm{f}_{2} / \mathrm{B}$ for a 2 -stage amplifier system with $N_{g 1} / N_{i}=$ $N_{g_{2}} / N_{i}=5 \mathrm{~dB}$ and $K_{1} / B=K_{2} / B=5 \mathrm{~dB}$. Preferred pairs $\left(\mathrm{f}_{1} / \mathrm{B}, \mathrm{f}_{2} / \mathrm{B}\right)$ are denoted as circles and the dash-dot line is the corresponding curve fit. 


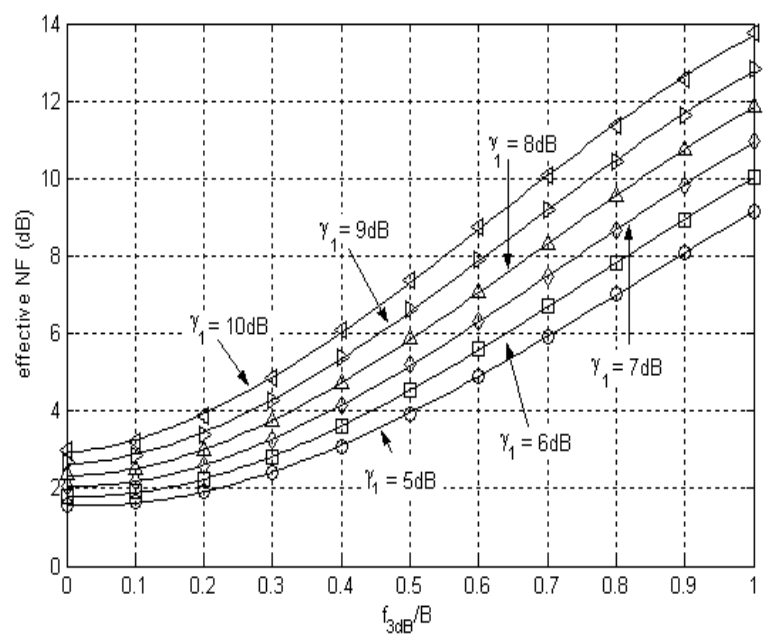

Fig. 5. The effective NF related to $f_{3 d B} / B$ and $\gamma_{1}$ for the 2-stage amplifier sytem, assuming $K_{1} / B=K_{2} / B=5 \mathrm{~dB}$.

Substituting (13) into (3) and integrating over the signal bandwidth, the effective NF can be obtained by numerically computing for

$$
F_{e f f}=B / \int_{0}^{B} \frac{1}{F_{s}(f)} d f
$$

Our design objective is to select $\left\{f_{1} / B, f_{2} / B, \ldots, f_{\mathrm{M}} / B\right\}$ given $\left\{\gamma_{1}, \gamma_{2}\right.$, $\ldots, \gamma_{M}$, so that the effective NF in (15) is optimally traded with the $M$-stage amplifier bandwidth of $f_{3 d B}$. This design objective is illustrated in Fig. 4 for a two-stage amplifier. Assuming $N_{g 1} / N_{i}=N_{g 2} / N_{i}$ $=5 \mathrm{~dB}$ and $K_{1} / B=K_{2} / B=5 \mathrm{~dB}$, Fig. 4 plots contours of constant effective $f_{3 d B} / B$ and effective NF as a function of $f_{1} / B$ and $f_{2} / B$. Solid contour lines closer to the origin represent decreasing effective NF values, whereas the dotted contour lines further away from the origin represent increasing effective $f_{3 d B} / B$ values. Since the amplifiers should be designed with $f_{1} / B$ and $f_{2} / B$ that maximize the effective $f_{3 d B} / B$ for a given effective NF, or equivalently, that minimize the effective NF for a given $f_{3 d B} / B$, the optimal design points, which are represented graphically using circles in Fig. 4, occur when the direction of the gradients of the effective $f_{3 d B} / B$ and NF contours are equal. More generally, these optimal design points for an $M$-stage amplifier can be determined by solving the following constrained optimization problem given a desired $f_{3 d B} / B$ value:

$$
\text { minimize } \quad F_{\text {eff }}
$$

$$
\text { subject to } \prod_{l=1}^{M}\left[f_{3 d B}^{2}+f_{l}^{2}\right]=2 \prod_{l=1}^{M} f_{l}^{2}
$$

where $F_{\text {eff }}$ is provided in (15). The optimization problem given in (16) can be solved numerically using iterative search techniques such as the sequential quadratic programming (SQP) method [7].

Fitting a quadratic curve through the optimal design points in our 2-stage amplifier example in Fig. 4, the relationship between the optimal $f_{1} / B$ and $f_{2} / B$ is given by

$$
\frac{f_{2}}{B}=2.5 \cdot \frac{f_{1}}{B}-1.3 \cdot\left(\frac{f_{1}}{B}\right)^{2}
$$

As is clear from (17), $f_{1} / B$ is smaller than $f_{2} / B$. This is because the noise associated with the first stage is more critical to the overall NF. Therefore, a narrower amplifier in the first stage to improve $F_{\text {eff }}$ followed by a wider amplifier achieves the minimum effective NF for a given $f_{3 d B}$. Generalizing this observation to an $M$-stage amplifier, the optimal design is to successively cascade the amplifier stages with widening bandwidths.

Based on the optimal design points, the effective NF can be plotted as a function of $f_{3 d B} / B$ as shown in Fig. 5 for our 2-stage amplifier example. This plot can be used to determine the optimal tradeoffs between the effective NF and $f_{3 d B} / B$ of the overall amplifier. Since the effective NF monotonically increases with increasing $f_{3 d B} / B$, the amplifiers can be designed with an $f_{3 d B}$ that corresponds to an effective NF that is slightly above (e.g., $1 \mathrm{~dB}$ ) the minimum effective NF.

\section{CONCLUSIONS}

For the NF of an amplifier to be a meaningful metric, the SNR at the input and output of the receiving system should measure the performance after the eventual digital decoding process, as it is ultimately the most relevant measure of performance. By defining the SNR as the MFB, the effective NF measures the degree of degradation in the achievable receiver performance caused by the receiving system.

The difficulty in amplifying the UWB signal stems from its bandwidth being a large fraction of the amplifier gain-bandwidth product. Hence, a design approach for maximizing the amplifier bandwidth for a given effective NF is described. Our analysis suggests that the optimal design is to successively cascade the amplifier stages with widening bandwidths.

\section{REFERENCES}

[1] M. Z. Win and R. A. Scholtz, "Impulse radio: How it works," IEEE Commun. Lett., vol. 2, no. 2, pp.36-38, Feb. 1998.

[2] M. Z. Win and R. A. Scholtz, "Ultra-wide bandwidth timehopping spread-spectrum impulse radio for wireless multiaccess communications," IEEE Trans. Commnu., vol. 48, no. 4, pp. 679-691, Apr. 2000.

[3] M. Z. Win and R. A. Scholtz, "On the roubustness of ultrawide bandwidth signals in dense multipath environments," IEEE Commun. Lett., vol. 2, no. 2, pp. 51-53, Feb. 1998.

[4] H. A. Haus, "IRE standards on methods of measuring noise in linear two ports, 1959," Proc. IRE, vol. 48, pp. 60-74, 1960.

[5] J. G. Proakis, Digital Communications, Boston: McGraw-Hill, 2001.

[6] H. T. Friis, "I. Noise figure of radio receivers," Proc. IRE, vol. 32, pp. 419-422, Jul. 1944.

[7] Goldfarb, D., "A family of variable metric updates derived by variational means," Mathematics of Computing., vol. 24, pp. 23-26. 1970 